\title{
Mechanistic Insights into Carbon-Carbon Coupling on NiAu and PdAu Single-Atom Alloys
}

Paul Kress ${ }^{\mathrm{a}}$, Romain Réocreux ${ }^{\mathrm{b}}$, Ryan Hannagan ${ }^{\mathrm{a}}$, Theodore Thuening ${ }^{\mathrm{a}}$, J. Anibal Boscoboinik ${ }^{\mathrm{c}}$, Michail Stamatakis ${ }^{\mathrm{b}^{*}}$ and E. Charles H. Sykes ${ }^{\mathrm{a}^{*}}$

${ }^{a}$ Department of Chemistry, Tufts University, Medford, Massachusetts 02155, United States.

${ }^{\mathrm{b}}$ Thomas Young Centre and Department of Chemical Engineering, University College London, Roberts Building, Torrington Place, London WC1E 7JE, United Kingdom.

${ }^{c}$ Brookhaven National Laboratory, Center for Functional Nanomaterials, Upton, NY 11973, United States.

\begin{abstract}
Carbon-carbon coupling is an important step in many catalytic reactions and performing $\mathrm{sp}^{3}-\mathrm{sp}^{3}$ carbon-carbon coupling heterogeneously is particularly challenging. It has been reported that PdAu single-atom alloy (SAA) model catalytic surfaces are able to selectively couple methyl groups producing ethane from methyl iodide. Herein, we extend this study to NiAu SAAs and find that $\mathrm{Ni}$ atoms in $\mathrm{Au}$ are active for $\mathrm{C}$-I cleavage and selective $\mathrm{sp}^{3}-\mathrm{sp}^{3}$ carbon-carbon coupling to produce ethane. Furthermore, we performed ab initio kinetic Monte Carlo simulations that include the effect of the iodine atom, which was previously considered a bystander species. We find that model NiAu surfaces exhibit a similar chemistry to PdAu, but the reason for the similarity is due to the role the iodine atoms play in terms of blocking the $\mathrm{Ni}$ atom active sites. Specifically, on $\mathrm{NiAu}$ SAAs the iodine atoms outcompete the methyl groups for occupancy of the Ni sites leaving the Me groups on $\mathrm{Au}$, while on PdAu SAAs, the binding strengths of methyl groups and iodine atoms at the Pd atom active site are more similar. These simulations shed light on the mechanism of this important $\mathrm{sp}^{3}$ - $\mathrm{sp}^{3}$ carbon-carbon coupling chemistry on SAAs. Furthermore, we discuss the effect of the iodine atoms on the reaction energetics and make an analogy between the effect of iodine as an active site blocker on this model heterogeneous catalyst and homogeneous catalysts in which ligands must detach in order for the active site to be accessed by the reactants.
\end{abstract}

Keywords: Würtz coupling, heterogeneous catalysis, STM, TPD, DFT, KMC 


\section{Introduction}

Carbon-carbon coupling is an important step in the upgrading of light alkanes which are becoming more abundant due to higher shale gas production rates. ${ }^{1}$ Performing selective $\mathrm{sp}^{3}-\mathrm{sp}^{3}$ carbon coupling is still very challenging despite its importance to many processes, for example in the production of pharmaceuticals. ${ }^{2,3}$ Typically, $\mathrm{sp}^{3}-\mathrm{sp}^{3}$ (Würtz) carbon coupling reactions are carried out using homogeneous catalysts; however, a number of issues plague homogeneous catalysis, such as difficulties in product-catalyst separation and catalyst deactivation over time. Therefore, there is interest in employing heterogeneous catalytic methods instead. Typically, Pd complexes are used as homogeneous catalysts and recently, a heterogeneous Pd-atom based catalyst with superior performance to homogeneous systems has been reported for Suzuki Coupling. ${ }^{4}$ Specific to this work, PdAu single-atom alloys (SAAs) investigated using a model single crystal approach, have been shown to perform selective $\mathrm{sp}^{3}-\mathrm{sp}^{3}$ carbon-carbon coupling when methyl iodide is used as the reactant. ${ }^{5}$ While $\mathrm{Pd}$ is a commonly used metal for coupling reactions, $\mathrm{Ni}$ is an attractive alternative of interest, in part, due to its lower cost.

In this work, model NiAu surfaces were studied with a range of surface science techniques to understand their structure and reactivity towards methyl iodine, while also making comparisons to previously investigated PdAu systems for the same reaction. NiAu and PdAu single-atom alloys were then modelled theoretically in order to understand the mechanism of $\mathrm{sp}^{3}-\mathrm{sp}^{3}$ carbon-carbon coupling on the two systems and the effect of the iodine in terms of its competition with methyl groups for the active sites. This synergistic approach, combining surface science and modelling techniques, has already been proven successful in elucidating structure-function relationships and delivering an atomic-scale understanding of coupling reaction mechanisms. ${ }^{6-11}$ Specifically, we combined Temperature Programmed Desorption (TPD), Reflection Absorption Infrared Spectroscopy (RAIRS), Scanning Tunneling Microscopy (STM), Density Functional Theory (DFT), and kinetic Monte Carlo (KMC) methods to first determine the model catalyst surface, then quantify the performance of the catalyst towards the coupling chemistry, and finally model these results with first-principles theory in order to get an atomic-scale picture of the reaction mechanism. Our results highlight the important role of iodine, which had previously been considered a bystander species, on the barrier for $\mathrm{sp}^{3}-\mathrm{sp}^{3}$ carbon coupling on PdAu SAA surfaces. 


\section{Methods}

All experiments were performed in one of three ultra-high vacuum chambers with base pressures below $2 \times 10^{-10}$ mbar. The $\mathrm{Au}(111)$ single crystals were cleaned by successive $\mathrm{Ar}^{+}$ sputtering cycles $(1.5 \mathrm{keV}, 10 \mu \mathrm{A})$ followed by annealing to $725 \mathrm{~K}$. Ni was deposited onto the Au(111) crystal held at $380 \mathrm{~K}$ using a Omicron Nanotechnology Focus EFM 3 electron beam evaporator and the Ni coverage was determined by XPS or CO TPD titration which has been previously reported. ${ }^{12}$ Liquid methyl iodide (Sigma Aldrich 99.5\%) was purified by freeze-pumpthaw cycles and was then dosed through a high-precision leak valve onto the crystal. TPD experiments were then performed in the UHV chamber using a Hiden quadruple mass spectrometer and a heating rate of $2 \mathrm{~K} \mathrm{~s}^{-1}$. STM studies were performed using a low-temperature scanning tunneling microscope (Omicron Nanotechnology) at $5 \mathrm{~K}$. Reflection absorption infrared spectroscopy (RAIRS) experiments were performed at the Center for Functional Nanomaterials $(\mathrm{CFN})$ at Brookhaven National Laboratory in a UHV system with a connected preparation chamber and X-ray photoelectron spectroscopy (XPS) chamber for measurement of the Ni content and cleanliness of sample. The XPS data was obtained at room temperature using a SPECS PHOIBOS NAP 150 hemispherical analyser and a monochromatic Al Ka X-ray source $(1486.6 \mathrm{eV}, \sim 0.25 \mathrm{eV}$ linewidth) focused on the sample to a spot size $<0.3 \mathrm{~mm}(0.05 \mathrm{eV}$ step, $0.1 \mathrm{~s}$ dwell time, 5 scans, $50 \mathrm{eV}$ pass energy). The infrared data was collected using a Bruker $80 \mathrm{~V}$ spectrometer with a polarizer and an MCT detector (2000 scans, $4 \mathrm{~cm}^{-1}$ resolution) in a UHV chamber with $\mathrm{KBr}$ windows and a beam to surface angle $\sim 8^{\circ}$. The IR spectra were taken while the chamber was filled to $1 \times 10^{-6}$ mbar CO.

The periodic Density Functional Theory (DFT) calculations were performed using the Vienna Ab initio Simulation package (VASP) 5.4.4. ${ }^{13-15}$ We used the nonlocal optB86b-vdW functional to describe the exchange-correlation potential. ${ }^{16-19}$ The core electrons were treated using the Projected Augmented Wave (PAW) method. ${ }^{20,21}$ The electronic density associated with the valence electrons was expanded on a plane wave basis set with an energy cut-off of $400 \mathrm{eV}$.

Two sets of slabs and Monkhorst-Pack ${ }^{22}$ k-point meshes were used:

- A 4-layer $\mathrm{p}(4 \times 4) \mathrm{Au}(111)$ slab and a $7 \times 7 \times 1 \mathrm{k}$-point mesh were used to describe the reactivity of gold sites. 
- A 4-layer $\mathrm{p}(3 \times 1) \mathrm{Au}(211) \mathrm{slab}$, where one of the step-edge Au atoms was substituted with $\mathrm{Ni}$ or $\mathrm{Pd}$, and a $11 \times 9 \times 1 \mathrm{k}$-point mesh were used to describe the reactivity of the dopant atom at the elbow of the herringbone reconstruction.

The slabs were built after optimizing the bulk structure of Au (lattice constant of $4.127 \AA$ ). Further optimizations of the surface structures were carried out after fixing the positions of the atoms of the first two layers at their bulk positions. Transition states were optimized using a combination of algorithms (CI-NEB, ${ }^{23,24}$ DIMER $^{24-26}$ and Quasi-Newton). Vibrational frequency calculations were performed for all species.

For a given elementary step the activation energy Ea is defined as the difference between the energy of the transition state $E_{T S}$ and the energy of the reactant(s) $E_{R}$, while the reaction energy $\Delta_{\mathrm{r}} \mathrm{E}$ is the difference between the energy of the product(s) $E_{\mathrm{P}}$ and that of the reactant(s) $E_{\mathrm{R}}$ :

$$
\begin{aligned}
& \mathrm{Ea}=\mathrm{E}_{\mathrm{TS}}-\mathrm{E}_{\mathrm{R}} \\
& \Delta_{\mathrm{r}} \mathrm{E}=\mathrm{E}_{\mathrm{P}}-\mathrm{E}_{\mathrm{R}}
\end{aligned}
$$

The Temperature Programmed Desorption (TPD) experiments were simulated within the graph-theoretical kinetic Monte Carlo (KMC) approach as implemented in Zacros 2.0. ${ }^{27,28}$ The kinetic constants were estimated within the approximations of harmonic Transition State Theory using parameters obtained from the DFT calculations (energies, frequencies and rotational constants). The reaction and energetic patterns are given in Figure S1. The simulations were run on periodic lattices comprising sites of four different types: top Au sites, top Pd (or Ni) sites, threefold Au sites, threefold PdAu (or NiAu) mixed sites.

The lattices were built ensuring a 3\% surface loading in Pd (or Ni) sites, randomly seeded as single atoms. Simulations on PdAu without iodine were run on a 15,300-site lattice $(10,200$ threefold sites and 5,100 top sites) as reported in our previous work on PdAu. All other simulations were performed on a 105,336-site lattice (35,112 top sites and 70,224 threefold sites) to ensure good sampling even under the effect of dopant site poisoning by iodine, which decreases the number of available dopant sites, the only active sites considered here for the coupling reaction. Without lateral interactions, each dopant atom could stabilize up to 6 iodine atoms on their mixed threefold sites; however, such configurations are not realistic in view of steric hindrance effects, due to the large size of the iodine atom. Therefore, to prevent such configurations from appearing in the KMC simulation, arbitrarily large lateral interactions were also added to the energetic model. 
In particular, we used an energy contribution of $1 \mathrm{eV}$ for the interaction between two iodine atoms adsorbed on two of the six mixed threefold sites of the same dopant. Each TPD trace was obtained by averaging the TPD signals of 10 simulations in order the reduce the noise due to the stochastic nature of the simulations.

\section{Results and Discussion}

Figure 1a shows a representative STM image of the NiAu SAA surface formed by depositing 0.02 ML Ni on a $\mathrm{Au}(111)$ surface at $380 \mathrm{~K}$ and cooling to $80 \mathrm{~K}$ for imaging. As has been reported before, the $\mathrm{Ni}$ atoms are seen alloyed into the Au surface in the regions around the elbows of the $\mathrm{Au}(111) 22 \times \sqrt{3}$ or "herringbone" reconstruction. ${ }^{29}$ These regions contain an edge dislocation that provides an entry site for the diffusing $\mathrm{Ni}$ atom to incorporate into the Au surface. 
In order to demonstrate that these $\mathrm{Ni}$ atom sites are isolated from one another in the $\mathrm{Au}$ surface we performed RAIRS of adsorbed $\mathrm{CO}$ as seen in Figure 1b. At low loadings (0.02
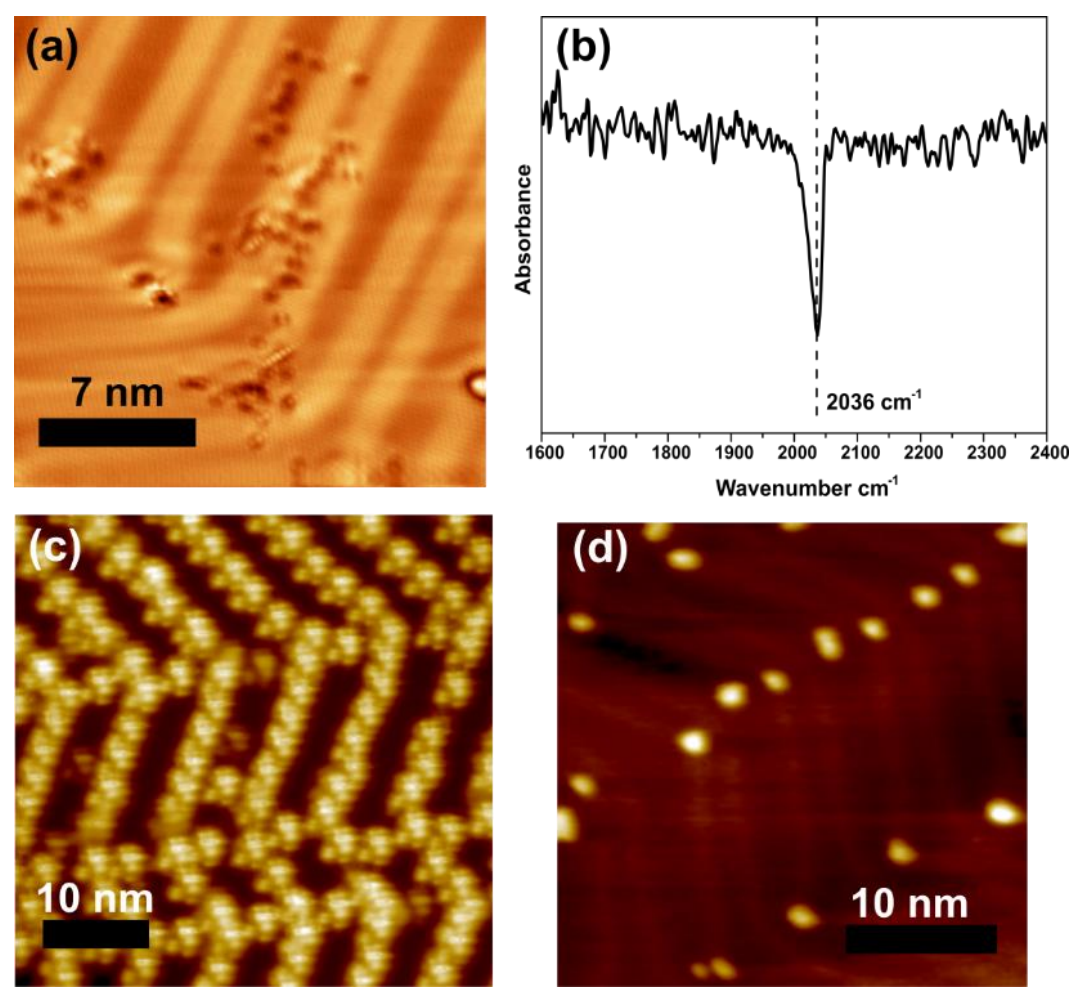

Figure 1: Characterization of the NiAu SAA surface and adsorption of the reactant methyl iodine (a) $80 \mathrm{~K} \mathrm{STM}$ image of as prepared $0.02 \mathrm{ML}$ NiAu SAA (b) CO RAIRs signal from 0.02 ML NiAu SAA (c) $5 \mathrm{~K}$ STM image of methyl iodide on a 0.02 ML NiAu SAA after a $100 \mathrm{~K}$ anneal to equilibrate the intact molecules (d) $5 \mathrm{~K}$ STM image of methyl iodide on 0.02 ML NiAu SAA surface after an anneal to $200 \mathrm{~K}$ that desorbs most of the molecules and cleaves the $\mathrm{C}$-I bond of those with access to a $\mathrm{Ni}$ atom site. monolayer (ML) Ni), only one sharp feature was observed at $2036 \mathrm{~cm}^{-1}$ consistent with the presence of isolated $\mathrm{Ni}$ atom sites, as no lower frequency signal due to multi-fold binding to $\mathrm{Ni}$ is seen. Previous RAIRS studies of CO at atop sites on $\mathrm{Ni}(111)$ have reported this feature at $2058 \mathrm{~cm}^{-1}$. The difference between this and our NiAu SAA result is due to the intrinsic electronic difference between isolated $\mathrm{Ni}$ sites vs. extended $\mathrm{Ni}(111) .{ }^{30-32}$ Further evidence for the lower frequency of the IR feature on the SAA comes from our DFT calculations that put this frequency at 1991 $\mathrm{cm}^{-1}$, which is reasonably accurate when taking into consideration inherent errors in DFT calculations.

After characterizing the structure of the NiAu SAA model surface we used TPD to study the reactivity of methyl groups on the surface. Due to the lack of $\mathrm{CH}_{4}$ reactivity in ultra-high 

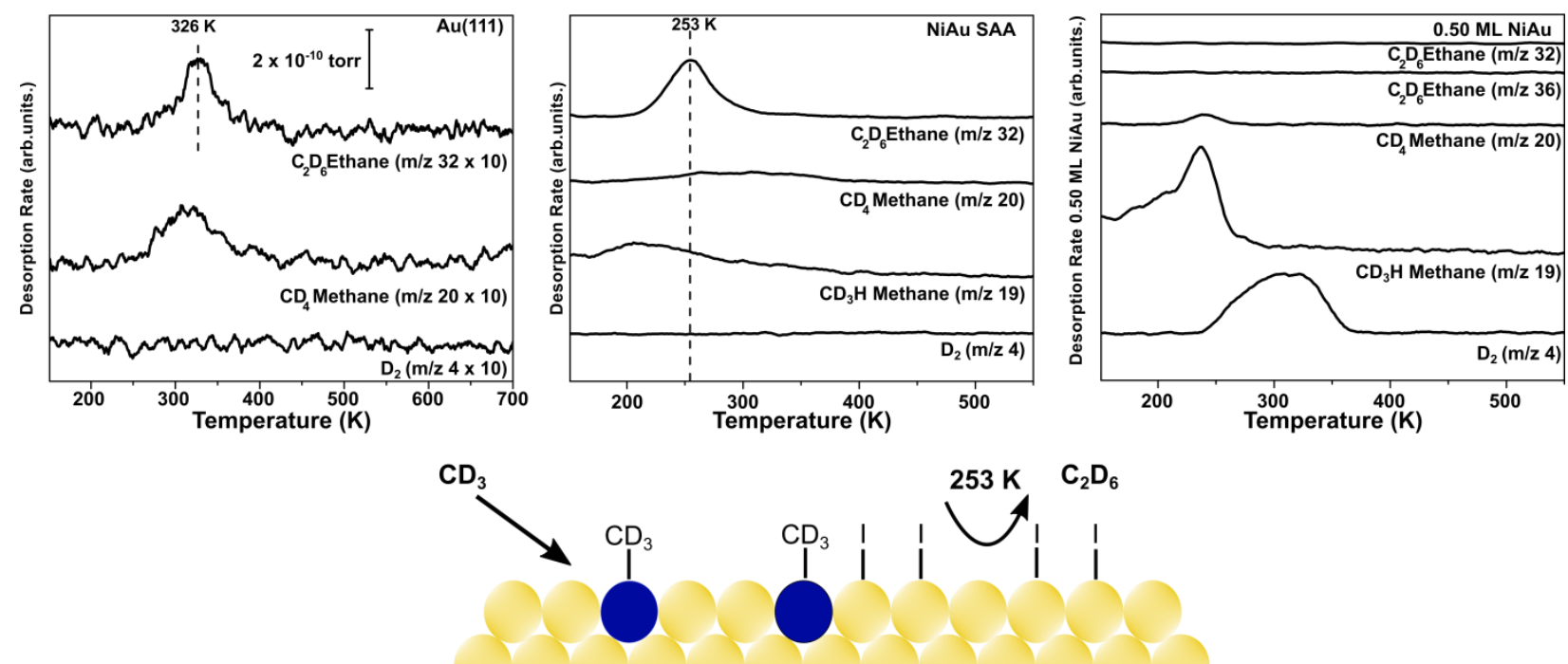

Figure 2: Reactivity of methyl iodine on $\mathrm{Au}(111)$ and NiAu model surfaces (a) TPD after deposition of 1 $\mathrm{L}$ (Langmuir) $\mathrm{CD}_{3} \mathrm{I}$ on pure $\mathrm{Au}(111)$ demonstrating the relative inertness of the $\mathrm{Au}(111)$ surface. Note that TPD traces on bare $\mathrm{Au}(111)$ are multiplied by a factor of 10 to make the desorption peaks visible, and that

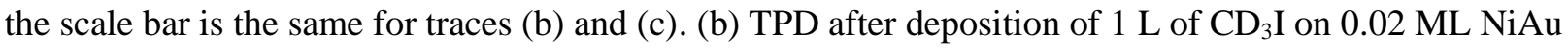
SAA showing ethane production and a small amount of methane produced from background hydrogenation of methyl groups. (c) TPD after deposition of $1 \mathrm{~L}$ of $\mathrm{CD}_{3} \mathrm{I}$ showing that on a surface with a higher Ni loading (0.5 ML Ni) the extended Ni sites lead to methyl decomposition and that the coupling pathway that leads to the formation of ethane is not in effect at this higher Ni loading.

vacuum experiments, we used methyl iodide to populate the NiAu SAA surface with methyl groups and study their coupling chemistry. STM measurements of methyl iodide molecules on the surface revealed that before the reaction, methyl iodide molecules are present at both the $\mathrm{Au}(111)$ herringbone reconstruction elbows where the $\mathrm{Ni}$ is present, as well as on the Au bare surface between these regions (Figure 1c). The linear ordering of methyl iodine molecules seen in this image is caused by the soliton walls of the herringbone reconstruction (seen in Figure 1a) that serve to corral the molecules between them. ${ }^{33}$ However, after an anneal to $200 \mathrm{~K}$ that removes unreacted methyl iodide from the surface, it can be seen that the only molecular species present on the surface are at the Ni sites (Figure 1d).

Figure 2 shows a series of TPD spectra that illustrate the reactivity of methyl iodide (MeI) on bare $\mathrm{Au}(111)$, a NiAu SAA surface, and higher coverage (0.5 ML) Ni on $\mathrm{Au}(111)$. On bare $\mathrm{Au}(111)$ the dissociation of the $\mathrm{C}$-I bond is difficult as evidenced by the fact that most of the MeI desorbs intact from the surface around $170 \mathrm{~K}$ before the C-I bond can be cleaved. However, small amounts of defects like step edges inherent to the $\mathrm{Au}(111)$ surface are able to dissociate the C-I 
bond and the resulting methyl groups couple and desorb as ethane, as seen by the small ethane desorption peak at $326 \mathrm{~K}$ in Figure $2 \mathrm{a} .{ }^{34}$ Note that the reactivity of the $\mathrm{Au}(111)$ is so low that the desorption traces in Figure 2a have been multiplied by a factor of 10 for clarity. A small methane peak around $320 \mathrm{~K}$ was also observed due to methyl radical rejection and subsequent hydrogenation on the chamber walls, in agreement with previous studies. ${ }^{34}$

In contrast to $\mathrm{Au}(111)$, when $0.03 \mathrm{ML}$ of $\mathrm{Ni}$ is added to the $\mathrm{Au}$ surface, a dramatic increase of reactivity is observed as shown in Figure $2 \mathrm{~b}$ and larger amounts of ethane are seen desorbing at $253 \mathrm{~K}$. At this tempearture temperature ethane desorption is limited by its formation on the surface and therefore its desorption temperature is representative of the activation barrier for ethane formation i.e. carbon-carbon coupling. Importantly, the much larger amounts of ethane evolved as compared to $\mathrm{Au}(111)$, and the fact that the ethane is formed around $80 \mathrm{~K}$ lower in temperature on the SAA compared to $\mathrm{Au}(111)$ reveal two important details. The first is that NiAu(111) SAAs facilitate low-temperature C-I cleavage that leads to a larger yield of ethane from the SAA vs. pure $\mathrm{Au}(111)$ from which most of the MeI desorbs from the surface around $170 \mathrm{~K}$ before it can react. Secondly, C-C coupling that leads to the formation of ethane occurs at much lower temperature on the NiAu SAA (250 K vs. $320 \mathrm{~K}$ from Au(111)) meaning that Ni atom sites also catalyze the coupling step of the reaction. This carbon-carbon coupling on the NiAu SAA surface is selective and there is no evidence of decomposition products from our TPD measurements. The only other species observed, other than ethane, was methane desorbing from both the $\mathrm{Au}(111)$ surface as mentioned, and the NiAu SAA. The use of isotopic labelling experiments enabled us to further investigate the origin of this methane. Specifically, $\mathrm{CD}_{3} \mathrm{I}$ was used to understand the role of background $\mathrm{H}_{2}$ found in all ultra-high vacuum chambers. Figure 2 shows that fully deuterated ethane $\left(\mathrm{C}_{2} \mathrm{D}_{6}\right)$ is the primary product desorbing from the NiAu SAA surface and that the methane produced is predominantly $\mathrm{CD}_{3} \mathrm{H}$. The production of $\mathrm{CD}_{3} \mathrm{H}$ has been observed before and is observed because of background $\mathrm{H}_{2}$ present in the chamber that is capable of hydrogenating adsorbed methyl groups. ${ }^{12,35-40}$

Furthermore, quantitative mass spectrometry was performed on the carbon species desorbing from the NiAu SAA surface and it was determined that there was a 1:1 relationship between $\mathrm{Ni}$ sites and carbon species desorbing as methane and ethane. This provides further evidence that the $\mathrm{Ni}$ atom sites are responsible for both $\mathrm{C}$-I bond cleavage, as evidenced by the 
larger amounts of ethane formed on NiAu SAAs vs. pure $\mathrm{Au}(111)$, and the $\mathrm{sp}^{3}-\mathrm{sp}^{3}$ carbon-carbon coupling reaction step, as evidenced by ethane formation at a temperature $\sim 75 \mathrm{~K}$ lower than pure $\mathrm{Au}(111)$.

In order to examine the reactivity of Ni ensembles in Au that are larger than one atom, we performed identical experiments on a $0.5 \mathrm{ML} \mathrm{NiAu}$ model surface as seen in Figure 2. Interestingly, on this surface, which contains extended Ni ensembles, the methyl groups produced from the dissociation of $\mathrm{CD}_{3} \mathrm{I}$ were not observed to undergo carbon-carbon coupling to produce ethane. Instead, they decomposed and underwent reaction with background hydrogen to produce deuterated methane $\mathrm{CD}_{3} \mathrm{H}$, as well as further decomposition that led to the desorption of $\mathrm{D}_{2}$ above room temperature. Together, these TPD results demonstrate that individual, isolated Ni atoms in $\mathrm{Au}(111)$ serve as active sites for both $\mathrm{C}$-I bond cleavage that initiates the reaction of methyl iodide, and for selective $\mathrm{sp}^{3}-\mathrm{sp}^{3}$ carbon-carbon coupling of surface-bound methyl groups to form ethane.

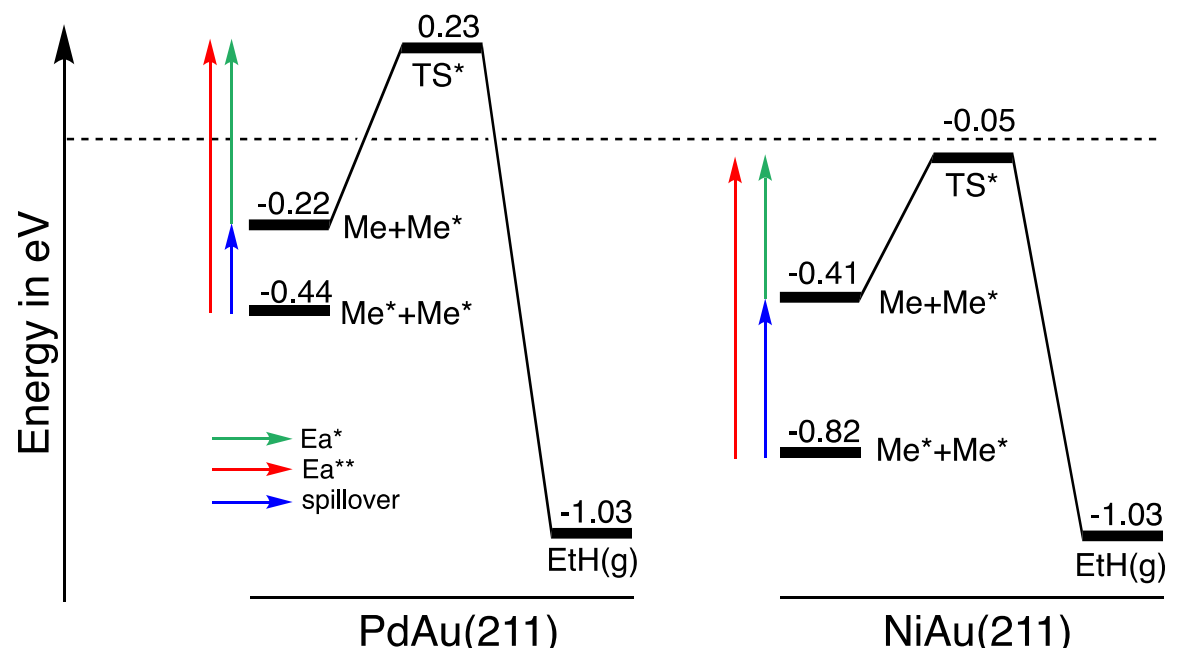

Figure 3. Energetics for the coupling of methyl groups on PdAu and NiAu SAA surfaces. In this energy diagram, the Pd and Ni single-atom sites are considered at the $\mathrm{Au}(211)$ facet and gold sites at the $\mathrm{Au}(111)$ facet. $\mathrm{Me}$ and $\mathrm{Me}^{*}$ represent methyl adsorbed on a gold top site and a single-atom top site respectively. Energies are referenced with respect to clean surfaces and methyl groups on gold top sites (Me).

In order to further understand the experimental results and also compare the energetics of the reaction steps to previously reported PdAu SAAs, we performed DFT and KMC simulations. The main challenge when modelling the reactivity of gold-based single-atom alloys is to describe the active site properly. As previously mentioned, the transition metals used as surface dopants $(\mathrm{Ni}, \mathrm{Pd})$, tend to alloy into the Au surface at the elbows of the herringbone reconstruction where 
edge dislocations are present, which have Au sites with a coordination number lower than 9. We showed in our previous work on PdAu SAAs that, while the (111) surface provides a good model for Au sites, the (211) surface is necessary to describe the reactivity of Pd sites. ${ }^{5}$ Similarly, we consider here that the Ni sites in the NiAu SAA are located at the (211) facet and that the Au sites are well described by the (111) surface. As with the PdAu SAA, the overall activation energy for the C-C coupling of methyl groups depends on the initial coverage and the associated representative initial state (see Figure 3 and $4 \mathrm{a}-\mathrm{c}$ ). For methyl group to dopant atom (Ni, Pd) ratios (denoted as $\sigma$ ) greater than 1 , all the dopant top sites are occupied by methyl groups (referred to as $\mathrm{Me}^{*}$ ) and excess methyl groups are adsorbed on gold sites (referred to as $\mathrm{Me}$ ) as represented in Figure 4b. For such coverages, the excess methyl groups at Au sites couple easily with dopantbound $\mathrm{Me}^{*}$ with a rather low activation energy Ea* of $0.36 \mathrm{eV}$ on $\mathrm{NiAu}$ and $0.45 \mathrm{eV}$ on $\mathrm{PdAu}$ (see barrier from $\mathrm{Me}+\mathrm{Me}^{*}$ in Figure 3). Now, for the case of $\sigma \leq 1$, all methyl species are adsorbed on dopant atom sites that are separated spatially from one another (as represented in Figure 4a) and the representative initial state for the coupling is $\mathrm{Me}^{*}+\mathrm{Me}^{*}$ (instead of the previously described $\left.\mathrm{Me}+\mathrm{Me}^{*}\right)$. For this situation, the two methyl groups ( $\mathrm{Me}^{*}$ ) cannot react readily since they are physically distanced. In order for the reaction to occur, one of the two methyl groups must spill over to gold $\left(\mathrm{Me}^{*} \rightarrow \mathrm{Me}\right)$ and then couple with a dopant-bound $\mathrm{Me}^{*}$. This spillover of methyl from the dopant to $\mathrm{Au}$ is endothermic (see Table 1) and significantly raises the activation energy (denoted by $\mathrm{Ea}^{* *}$ ) to $0.77 \mathrm{eV}$ on $\mathrm{NiAu}$ and $0.67 \mathrm{eV}$ on $\mathrm{PdAu}$ (compare red and green arrows in Figure 3).

Table 1. Reaction energies for the spillover of methyl and iodine species from the dopant atom sites to the host $\mathrm{Au}(111)$ metal surface and the exchange between iodine and methyl species adsorbed at the dopant sites on PdAu and NiAu SAA. Starred $\left(^{*}\right)$ adsorbates are bound to the dopant (which is considered at the step-edge of the (211) facet) and unstarred adsorbates are located on the $\mathrm{Au}(111)$ surface. The overall exchange process is the difference between the two elementary steps. Energies are given in $\mathrm{eV}$.

\begin{tabular}{|l|c|c|}
\hline & PdAu SAA & NiAu SAA \\
\hline Spillover elementary steps & 0.22 & 0.41 \\
$\mathrm{Me}^{*} \rightarrow \mathrm{Me}$ & 0.36 & 0.62 \\
$\mathrm{I}^{*} \rightarrow \mathrm{I}$ & & \\
Overall exchange process & 0.14 & 0.21 \\
$\mathrm{I}^{*}+\mathrm{Me} \rightarrow \mathrm{I}+\mathrm{Me}^{*}$ & \\
\hline
\end{tabular}


(a) initial state for $\sigma=1,[1]=0$

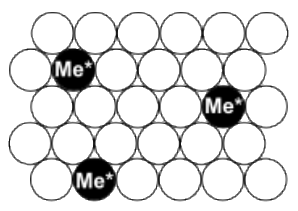

(b) initial state for $\sigma=2,[l]=0$

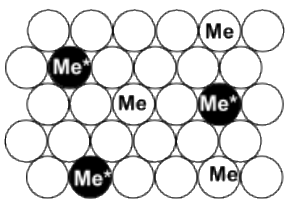

(c) initial state for $\sigma=1,[\mathrm{l}]=[\mathrm{Me}]$

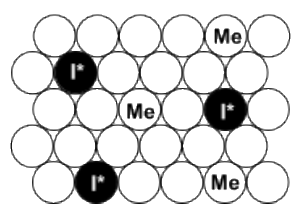

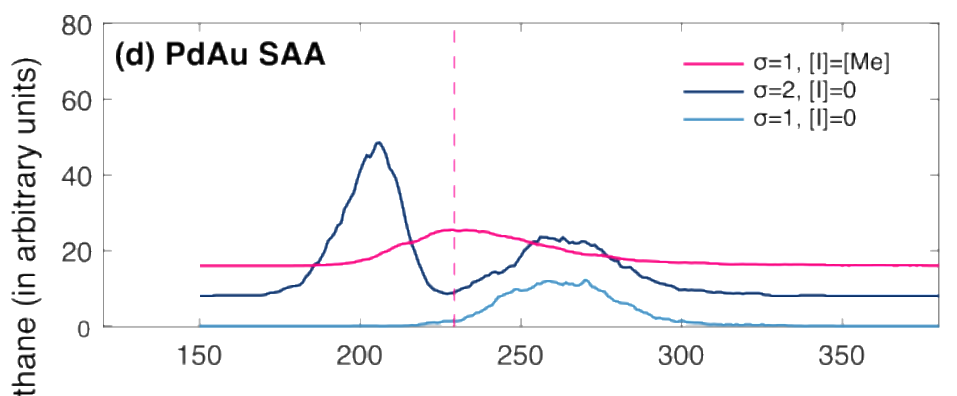

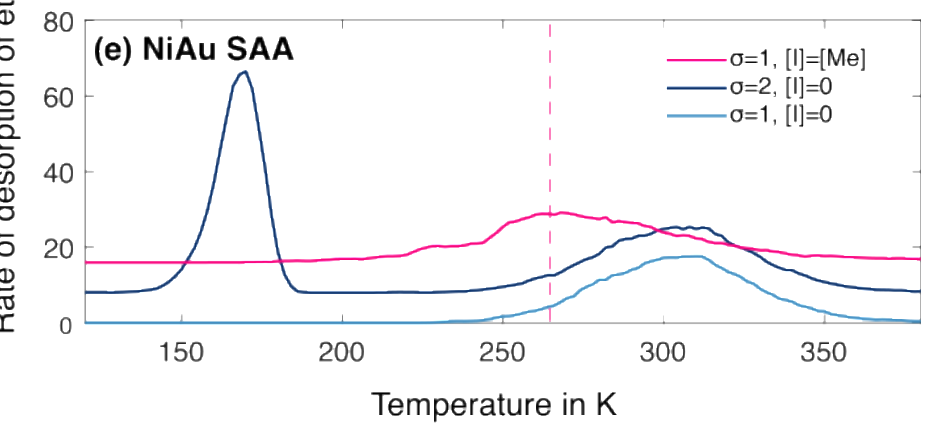

Figure 4. KMC-simulated TPD traces using the parameters computed at the DFT level. Different initial conditions were simulated (a-c). The traces correspond to the production of ethane on (d) PdAu SAA and (e) NiAu SAA. The dashed line indicates the position of the temperature peak when iodine is explicitly taken into account in the KMC simulations. The temperature ramps used in the simulations are as follows: $2 \mathrm{~K} / \mathrm{s}$ for $\mathrm{NiAu}$ and $1 \mathrm{~K} / \mathrm{s}$ for PdAu, consistent with experimental TPDs.

We then performed KMC simulations to directly compare the experimental TPDs with simulated TPDs based on the DFT calculations. We first discuss the case in which the effect of iodine is neglected. On both PdAu and NiAu SAAs, the KMC simulated TPDs show two ethane desorption peaks (dark blue traces in Figure 4d-e). The first one at low temperature (200 K for $\mathrm{PdAu}$ and $160 \mathrm{~K}$ for $\mathrm{NiAu})$ is only seen for methyl group to dopant atom ratios $(\sigma)>1$, and the second ethane desorption peak at higher temperature ( $255 \mathrm{~K}$ for PdAu and $310 \mathrm{~K}$ for $\mathrm{NiAu}$ ) appears for values of $\sigma$ above and below 1. This is in line with the coverage dependent activation energies (Ea* and $\mathrm{Ea}^{* *}$ ) expected from our DFT calculations. Experimentally, $\sigma \sim 1$ and only one peak is seen around $250 \mathrm{~K}$. This is why, in our previous work on the PdAu SAA, we had attributed this peak to the coupling of two methyls initially distanced on two different dopant sites (initial state $\mathrm{Me}^{*}+\mathrm{Me}^{*}$, see Figure 4a). In the case of NiAu SAA, this simulated peak shifts to $310 \mathrm{~K}$, in poor agreement with experimental data (see Figure 2). This discrepancy between our experimental and theoretical results suggests that iodine, which is produced via the dissociated adsorption of methyl 
iodide and often thought to be a bystander, may actually play a role on the carbon-carbon coupling energetics.

Our DFT calculations indicate that the dopant sites stabilize iodine atoms to a larger extent than methyl groups as can been seen in Table 1. Therefore, when the systems are in a representative low-temperature initial state, all dopant sites should be occupied by iodine (I*) and all methyl groups should reside on gold sites (Me), as illustrated in Figure 4c. As the dopant is the active site for the coupling, the first step of the coupling reaction is the endothermic exchange of iodine with methyl $\left(\mathrm{I}^{*}+\mathrm{Me} \rightarrow \mathrm{I}+\mathrm{Me} *\right.$ in Table 1$)$. From this state, transient dopant-bound Me* can couple with a methyl group bound to gold. This brings the overall activation energy for carbon-carbon coupling to $0.59 \mathrm{eV}$ for $\mathrm{PdAu}$ and $0.57 \mathrm{eV}$ for $\mathrm{NiAu}$. When taking this exchange equilibrium into account in the KMC simulations, the TPD traces show a broad peak at $235 \mathrm{~K}$ for PdAu and $260 \mathrm{~K}$ for NiAu (pink traces in Figure 4), in close agreement with experimental data (see Figure 2).

In the absence of iodine, our simulations have evidenced two regimes for the $\mathrm{C}-\mathrm{C}$ coupling (see Figures 4d-e and S2). When there is an excess of methyl, the dopant sites act as very efficient active sites. Dopant-bound methyls couple with excess methyls on gold sites at very low temperatures. When the methyl to dopant ratio becomes stoichiometric or sub-stoichiometric, every methyl is stabilized on physically distanced dopant sites. Hence, they need to overcome the thermodynamic barrier to spill over to gold and become reactive. In this regime, where there are more active sites than reactants, the reactant itself hinders the coupling and the latter can only happen at higher temperatures. This widens the temperature range under which methyls are present on the surface and may open the possibility for other undesired reactions to happen. When iodine is added in stoichiometric proportion with respect to the dopant, it pushes methyl onto gold, where it does not undergo any reactions. However, methyl groups and iodine atoms can transiently exchange, giving rise to a configuration that is particularly reactive towards the coupling to ethane. Because iodine poisons the active site to some extent, the low-temperature regime coupling occurs at a moderate temperature when iodine is present. Most importantly, these simulations indicate that when iodine is present, methyl groups never get trapped on the dopant site where they can only couple at a higher temperature.

While this situation of competitive binding of iodine atoms to the active sites for methyl group coupling complicates the mechanistic picture of how the coupling reaction occurs, it allows 
us to make an interesting comparison to common, and very well understood, homogenously catalyzed coupling reactions. Specifically, homogeneous catalysts are typically metal centers surrounded by ligands (L) as seen in Figure 5. In order for a coupling reaction to proceed, one or two ligands must detach from the metal center so that the reactants (blue circles) bind to the metal center. The reactants subsequently couple and desorb from the metal complex and the ligands are replaced. This is essentially the same mechanism that we have discovered for heterogeneous carbon-carbon coupling on NiAu and PdAu SAAs as shown in Figure 5. In an analogous manner the iodine "ligand" bound to the metal atom dopant must be exchanged with a methyl group that can then couple with a second methyl group supplied by the Au surface, and after the coupling is complete and ethane desorbs the metal center is again occupied by iodine.
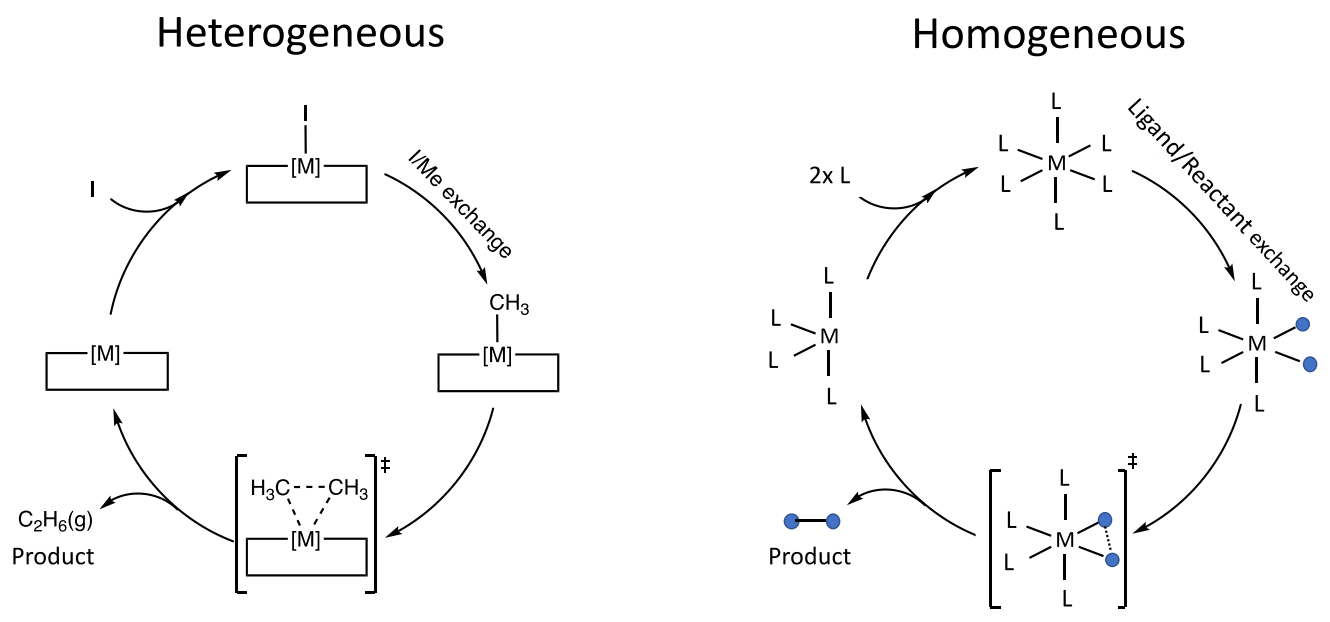

Figure 5. Comparing the mechanism of carbon-carbon coupling on SAA surfaces (heterogeneous) with a typical homogenously catalyzed coupling mechanism. In the heterogeneous catalytic cycle, adsorbates are exchanged between the active site $\mathrm{M}$ ( $\mathrm{Pd}$ or $\mathrm{Ni}$ ) and the non-reactive Au sites. In the homogeneous catalytic cycles, the species are exchanged between the active site $\mathrm{M}$ and the solvent.

\section{Conclusions}

Our experiments reveal that NiAu SAA surfaces, like PdAu SAAs, are able to catalyze $\mathrm{sp}^{3}-\mathrm{sp}^{3}$ carbon-carbon coupling of surface-bound methyl groups to form ethane. Unlike clusters of Ni or Pd (observed in alloys at higher dopant coverages) that are incapable of ethane formation and instead catalyze decomposition of the methyl groups, single Ni or Pd atoms catalyze both C-I 
cleavage in the reactant molecule and carbon-carbon coupling. The latter occurs at lower temperatures in the $\mathrm{NiAu}(111) \mathrm{SAA}$ than on pure $\mathrm{Au}(111)$, providing evidence that the reaction indeed occurs at the $\mathrm{Ni}$ atom sites. While the iodine atom produced by C-I cleavage in methyl iodide is often thought to be a bystander in such coupling reactions, we treat it explicitly in our DFT calculations and find that surface-bound iodine atoms outcompete surface-bound methyl groups for binding to the $\mathrm{Ni}$ or $\mathrm{Pd}$ atom sites in $\mathrm{Au}$. This effect is particularly pronounced for the $\mathrm{NiAu}$ SAA and we find that the energy to displace the iodine atom from the $\mathrm{Ni}$ active site must be considered in order to accurately model the experimental results and reproduce the correct desorption temperatures with $\mathrm{KMC}$. This is analogous to the mechanism of coupling reactions on homogeneous catalysts in which ligands must detach from the active metal center in order for the reactants to be activated and the coupling product formed.

\section{Acknowledgements}

This work was supported as part of Integrated Mesoscale Architectures for Sustainable Catalysis, an Energy Frontier Research Center funded by the U.S. Department of Energy, Office of Science, Office of Basic Energy Sciences under Award DE-SC0012573. R.R. was funded by the Leverhulme Trust (Grant No. RPG-2018-209). Part of the research used resources at the Center for Functional Nanomaterials, Brookhaven National Laboratory, supported by the U.S. Department of Energy, Office of Basic Energy Sciences, under Contract No. DE-SC0012704. We are also grateful to the UK Materials and Molecular Modelling Hub, which is partially funded by EPSRC (EP/P020194/1), for computational resources.

\section{Data Availability}

The data that support the findings of this study are available from the corresponding author upon reasonable request.

\section{References}

(1) Galadima, A.; Muraza, O. Revisiting the Oxidative Coupling of Methane to Ethylene in the Golden Period of Shale Gas: A Review. J. Ind. Eng. Chem. 2016, 37, 1-13. https://doi.org/10.1016/j.jiec.2016.03.027.

(2) Yin, L.; Liebscher, J. Carbon-Carbon Coupling Reactions Catalyzed by Heterogeneous 
Palladium Catalysts. Chem. Rev. 2007, 107 (1), 133-173.

https://doi.org/10.1021/cr0505674.

(3) Phan, N. T. S.; Van Der Sluys, M.; Jones, C. W. On the Nature of the Active Species in Palladium Catalyzed Mizoroki-Heck and Suzuki-Miyaura Couplings - Homogeneous or Heterogeneous Catalysis, a Critical Review. Adv. Synth. Catal. 2006, 348 (6), 609-679. https://doi.org/10.1002/adsc.200505473.

(4) Chen, Z.; Vorobyeva, E.; Mitchell, S.; Fako, E.; Ortuño, M. A.; López, N.; Collins, S. M.; Midgley, P. A.; Richard, S.; Vilé, G.; Pérez-Ramírez, J. A Heterogeneous Single-Atom Palladium Catalyst Surpassing Homogeneous Systems for Suzuki Coupling. Nat. Nanotechnol. 2018, 13 (August), 1-6. https://doi.org/10.1038/s41565-018-0167-2.

(5) Réocreux, R.; Uhlman, M.; Thuening, T.; Kress, P.; Hannagan, R.; Stamatakis, M.; Sykes, E. C. H. Efficient and Selective Carbon-Carbon Coupling on Coke-Resistant PdAu SingleAtom Alloys. Chem. Commun. 2019, 55 (100), 15085-15088. https://doi.org/10.1039/c9cc07932g.

(6) Xi, M.; Bent, B. E. Mechanisms of the Ullmann Coupling Reaction in Adsorbed Monolayers. J. Am. Chem. Soc. 1993, 115 (16), 7426-7433. https://doi.org/10.1021/ja00069a048.

(7) Fan, Q.; Gottfried, J. M.; Zhu, J. Surface-Catalyzed C-C Covalent Coupling Strategies toward the Synthesis of Low-Dimensional Carbon-Based Nanostructures. Acc. Chem. Res. 2015, 48 (8), 2484-2494. https://doi.org/10.1021/acs.accounts.5b00168.

(8) Fairbrother, D. H.; Peng, X. D.; Viswanathan, R.; Stair, P. C.; Trenary, M.; Fan, J. Carbon-Carbon Coupling of Methyl Groups on Pt(111). Surf. Sci. 1993, 285 (1-2). https://doi.org/10.1016/0039-6028(93)90900-5.

(9) Kanuru, V. K.; Kyriakou, G.; Beaumont, S. K.; Papageorgiou, A. C.; Watson, D. J.; Lambert, R. M. Sonogashira Coupling on an Extended Gold Surface in Vacuo: Reaction of Phenylacetylene with Iodobenzene on Au(111). J. Am. Chem. Soc. 2010, 132 (23), 8081-8086. https://doi.org/10.1021/ja1011542.

(10) Borkowski, T.; Trzeciak, A. M.; Bukowski, W.; Bukowska, A.; Tylus, W.; Kepiński, L. Palladium(0) Nanoparticles Formed in Situ in the Suzuki-Miyaura Reaction: The Effect of a Palladium(II) Precursor. Appl. Catal. A Gen. 2010, 378 (1), 83-89. https://doi.org/10.1016/j.apcata.2010.02.004.

(11) Paul, A.; Michael, X.; Bent, B. E. Disproportionation and Coupling Reactions of Alkyl Iodides on a $\mathrm{Au}(111)$ Surface. 1993.

(12) Lucci, F. R.; Darby, M. T.; Mattera, M. F. G.; Ivimey, C. J.; Therrien, A. J.; Michaelides, A.; Stamatakis, M.; Sykes, E. C. H. Controlling Hydrogen Activation, Spillover, and Desorption with Pd-Au Single-Atom Alloys. J. Phys. Chem. Lett. 2016, 7 (3), 480-485. https://doi.org/10.1021/acs.jpclett.5b02400.

(13) Kresse, G.; Hafner, J. Ab Initio Molecular Dynamics for Liquid Metals. Phys. Rev. B 
1993, 47, 558-561. https://doi.org/10.1103/PhysRevB.47.558.

(14) Kresse, G.; Furthmüller, J. Efficient Iterative Schemes for Ab Initio Total-Energy Calculations Using a Plane-Wave Basis Set. Phys. Rev. B 1996, 54, 11169-11186. https://doi.org/10.1103/PhysRevB.54.11169.

(15) Kresse, G.; Furthmüller, J. Efficiency of Ab-Initio Total Energy Calculations for Metals and Semiconductors Using a Plane-Wave Basis Set. Comput. Mater. Sci. 1996, 6 (1), 1550. https://doi.org/10.1016/0927-0256(96)00008-0.

(16) Dion, M.; Rydberg, H.; Schröder, E.; Langreth, D. C.; Lundqvist, B. I. Van Der Waals Density Functional for General Geometries. Phys. Rev. Lett. 2004, 92, 246401. https://doi.org/10.1103/PhysRevLett.92.246401.

(17) Klimeš, J.; Bowler, D. R.; Michaelides, A. Chemical Accuracy for the van Der Waals Density Functional. J. Phys. Condens. Matter 2010, 22, 022201. https://doi.org/10.1088/0953-8984/22/2/022201.

(18) Klimeš, J.; Bowler, D. R.; Michaelides, A. Van Der Waals Density Functionals Applied to Solids. Phys. Rev. B - Condens. Matter Mater. Phys. 2011, 83 (19), 1-13. https://doi.org/10.1103/PhysRevB.83.195131.

(19) Klimeš, J.; Michaelides, A. Perspective: Advances and Challenges in Treating van Der Waals Dispersion Forces in Density Functional Theory. J. Chem. Phys. 2012, 137, 120901-120913. https://doi.org/10.1063/1.4754130.

(20) Blöchl, P. Projector Augmented-Wave Method. 1994, 50, 17953.

(21) Kresse, G.; Joubert, D. From Ultrasoft Pseudopotentials to the Projector AugmentedWave Method. Phys. Rev. B 1999, 59, 1758-1775. https://doi.org/10.1103/PhysRevB.59.1758.

(22) Monkhorst, H. J.; Pack, J. D. Special Points for Brillouin-Zone Integrations. Phys. Rev. B 1976, 13, 5188-5192. https://doi.org/10.1103/PhysRevB.13.5188.

(23) Sheppard, D.; Terrell, R.; Henkelman, G. Optimization Methods for Finding Minimum Energy Paths. J. Chem. Phys. 2008, 128, 134106. https://doi.org/10.1063/1.2841941.

(24) Henkelman, G.; Uberuaga, B. P.; Jónsson, H. A Climbing Image Nudged Elastic Band Method for Finding Saddle Points and Minimum Energy Paths. J. Chem. Phys. 2000, 113, 9901. https://doi.org/10.1063/1.1329672.

(25) Heyden, A.; Bell, A. T.; Keil, F. J. Efficient Methods for Finding Transition States in Chemical Reactions: Comparison of Improved Dimer Method and Partitioned Rational Function Optimization Method. J. Chem. Phys. 2005, 123, 224101. https://doi.org/10.1063/1.2104507.

(26) Henkelman, G.; Jónsson, H. A Dimer Method for Finding Saddle Points on High Dimensional Potential Surfaces Using Only First Derivatives. J. Chem. Phys. 1999, 111, 7010. https://doi.org/10.1063/1.480097. 
(27) Stamatakis, M.; Vlachos, D. G. A Graph-Theoretical Kinetic Monte Carlo Framework for on-Lattice Chemical Kinetics. J. Chem. Phys. 2011, 134 (21), 214115.

https://doi.org/10.1063/1.3596751.

(28) Nielsen, J.; D’Avezac, M.; Hetherington, J.; Stamatakis, M. Parallel Kinetic Monte Carlo Simulation Framework Incorporating Accurate Models of Adsorbate Lateral Interactions. J. Chem. Phys. 2013, 139 (22), 224706. https://doi.org/10.1063/1.4840395.

(29) Wang, Z.-T.; Darby, M. T.; Therrien, A. J.; El-Soda, M.; Michaelides, A.; Stamatakis, M.; Sykes, E. C. H. Preparation, Structure, and Surface Chemistry of Ni-Au Single Atom Alloys. J. Phys. Chem. C 2016, 120 (25), 13574-13580. https://doi.org/10.1021/acs.jpcc.6b03473.

(30) Kruppe, C. M.; Krooswyk, J. D.; Trenary, M. Polarization-Dependent Infrared Spectroscopy of Adsorbed Carbon Monoxide to Probe the Surface of a $\mathrm{Pd} / \mathrm{Cu}(111)$ Single-Atom Alloy. J. Phys. Chem. C 2017, 121 (17), 9361-9369. https://doi.org/10.1021/acs.jpcc.7b01227.

(31) Patel, D. A.; Hannagan, R. T.; Kress, P. L.; Schilling, A. C.; Çlnar, V.; Sykes, E. C. H. Atomic-Scale Surface Structure and CO Tolerance of NiCu Single-Atom Alloys. J. Phys. Chem. C 2019, 123 (46), 28142-28147. https://doi.org/10.1021/acs.jpcc.9b07513.

(32) Hannagan, R. T.; Patel, D. A.; Cramer, L. A.; Schilling, A. C.; Ryan, P. T. P.; Larson, A. M.; Çınar, V.; Wang, Y.; Balema, T. A.; Sykes, E. C. H. Combining STM, RAIRS and TPD to Decipher the Dispersion and Interactions Between Active Sites in RhCu SingleAtom Alloys. ChemCatChem 2020, 12 (2), 488-493. https://doi.org/10.1002/cctc.201901488.

(33) Sykes, E. C. H.; Mantooth, B. A.; Han, P.; Donhauser, Z. J.; Weiss, P. S. SubstrateMediated Intermolecular Interactions: A Quantitative Single Molecule Analysis. J. Am. Chem. Soc. 2005, 127 (19), 7255-7260. https://doi.org/10.1021/ja0472331.

(34) Paul, Anumita; Bent, B. Alkyl Coupling on Copper, Silver, and Gold: Correlation between the Coupling Rate and the Metal-Alkyl Bond Strength. J. Catal. 1994, 264-271.

(35) Darby, M. T.; Stamatakis, M.; Michaelides, A.; Sykes, E. C. H. Lonely Atoms with Special Gifts: Breaking Linear Scaling Relationships in Heterogeneous Catalysis with Single-Atom Alloys. J. Phys. Chem. Lett. 2018, acs.jpclett.8b01888. https://doi.org/10.1021/acs.jpclett.8b01888.

(36) Lucci, F. R.; Marcinkowski, M. D.; Lawton, T. J.; Sykes, E. C. H. H2 Activation and Spillover on Catalytically Relevant Pt-Cu Single Atom Alloys. J. Phys. Chem. C 2015, 119 (43), 24351-24357. https://doi.org/10.1021/acs.jpcc.5b05562.

(37) Aich, P.; Wei, H.; Basan, B.; Kropf, A. J.; Schweitzer, N. M.; Marshall, C. L.; Miller, J. T.; Meyer, R. Single-Atom Alloy Pd-Ag Catalyst for Selective Hydrogenation of Acrolein. J. Phys. Chem. C 2015, 119 (32), 18140-18148.

https://doi.org/10.1021/acs.jpcc.5b01357. 
(38) Pei, G. X.; Liu, X. Y.; Wang, A.; Lee, A. F.; Isaacs, M. A.; Li, L.; Pan, X.; Yang, X.; Wang, X.; Tai, Z.; Wilson, K.; Zhang, T. Ag Alloyed Pd Single-Atom Catalysts for Efficient Selective Hydrogenation of Acetylene to Ethylene in Excess Ethylene. ACS Catal. 2015, 5 (6), 3717-3725. https://doi.org/10.1021/acscatal.5b00700.

(39) Marcinkowski, M. D.; Jewell, A. D.; Stamatakis, M.; Boucher, M. B.; Lewis, E. A.; Murphy, C. J.; Kyriakou, G.; Sykes, E. C. H. Controlling a Spillover Pathway with the Molecular Cork Effect. Nat. Mater. 2013, 12 (6), 523-528. https://doi.org/10.1038/nmat3620.

(40) Kyriakou, G.; Boucher, M. B.; Jewell, A. D.; Lewis, E. A.; Lawton, T. J.; Baber, A. E.; Tierney, H. L.; Flytzani-Stephanopoulos, M.; Sykes, E. C. H. Isolated Metal Atom Geometries as a Strategy for Selective Heterogeneous Hydrogenations. Science (80-. ). 2012, 335 (6073), 1209-1212. https://doi.org/10.1126/science.1215864. 


\section{Supplemental Information}

\section{Mechanistic Insights into Carbon-Carbon Coupling on $\mathrm{NiAu}$ and PdAu Single-Atom Alloys}

Paul Kress ${ }^{\mathrm{a}}$, Romain Réocreux ${ }^{\mathrm{b}}$, Ryan Hannagan ${ }^{\mathrm{a}}$, Theodore Thuening ${ }^{\mathrm{a}}$, J. Anibal Boscoboinik ${ }^{\mathrm{c}}$, Michail Stamatakis ${ }^{\mathrm{b}^{*}}$ and E. Charles H. Sykes ${ }^{\mathrm{a}^{*}}$

${ }^{a}$ Department of Chemistry, Tufts University, Medford, Massachusetts 02155, United States.

${ }^{\mathrm{b}}$ Thomas Young Centre and Department of Chemical Engineering, University College London, Roberts Building, Torrington Place, London WC1E 7JE, United Kingdom.

${ }^{\mathrm{c}}$ Brookhaven National Laboratory, Center for Functional Nanomaterials, Upton, NY 11973, United States. 

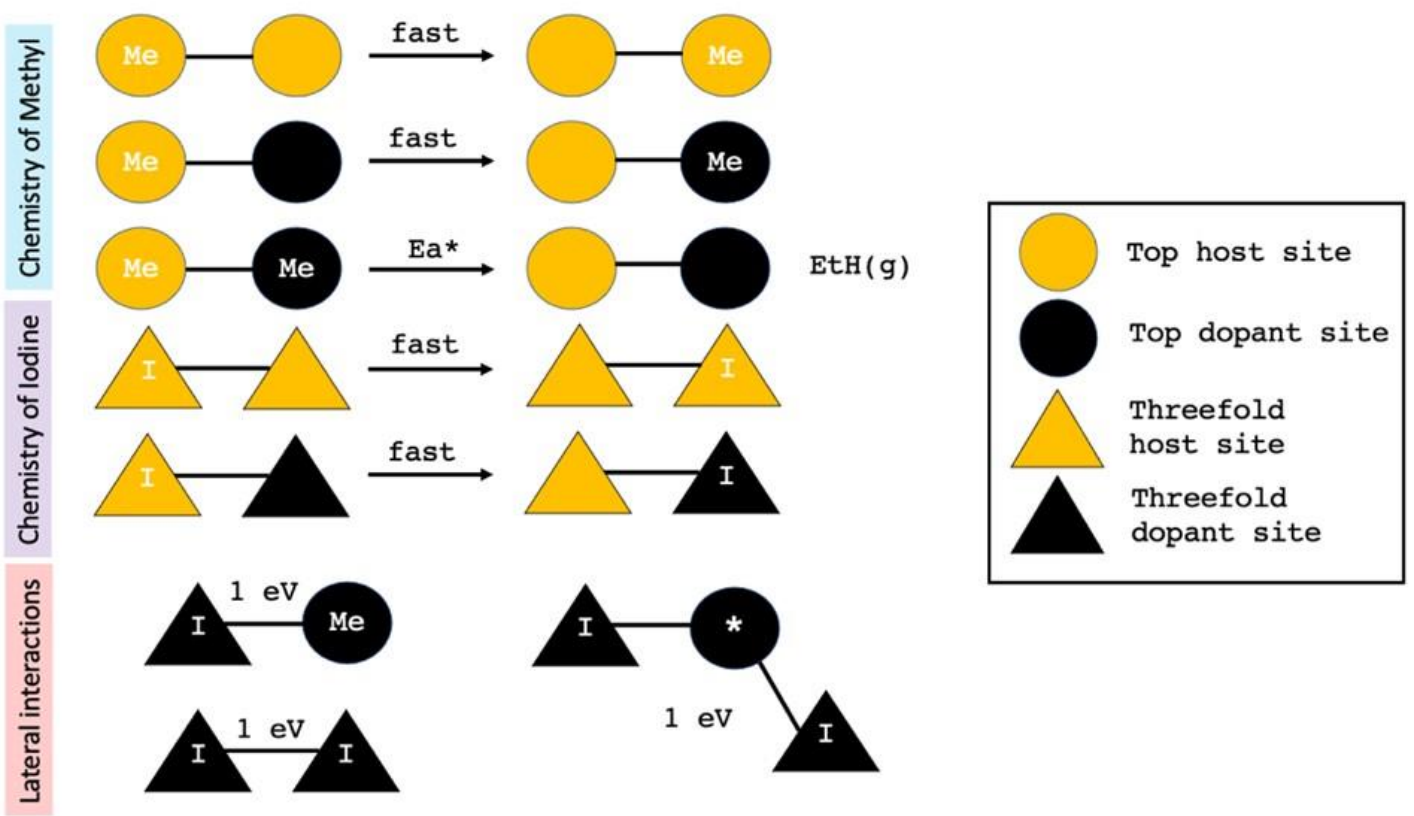

Figure S1. Reaction and energetic interaction patterns used in the graph theoretical KMC simulations. Ea* is defined in the main text. Lateral interactions for configurations that are not stable according to our DFT calculations were arbitrarily set to $1 \mathrm{eV}$ to make them unlikely during the simulations.
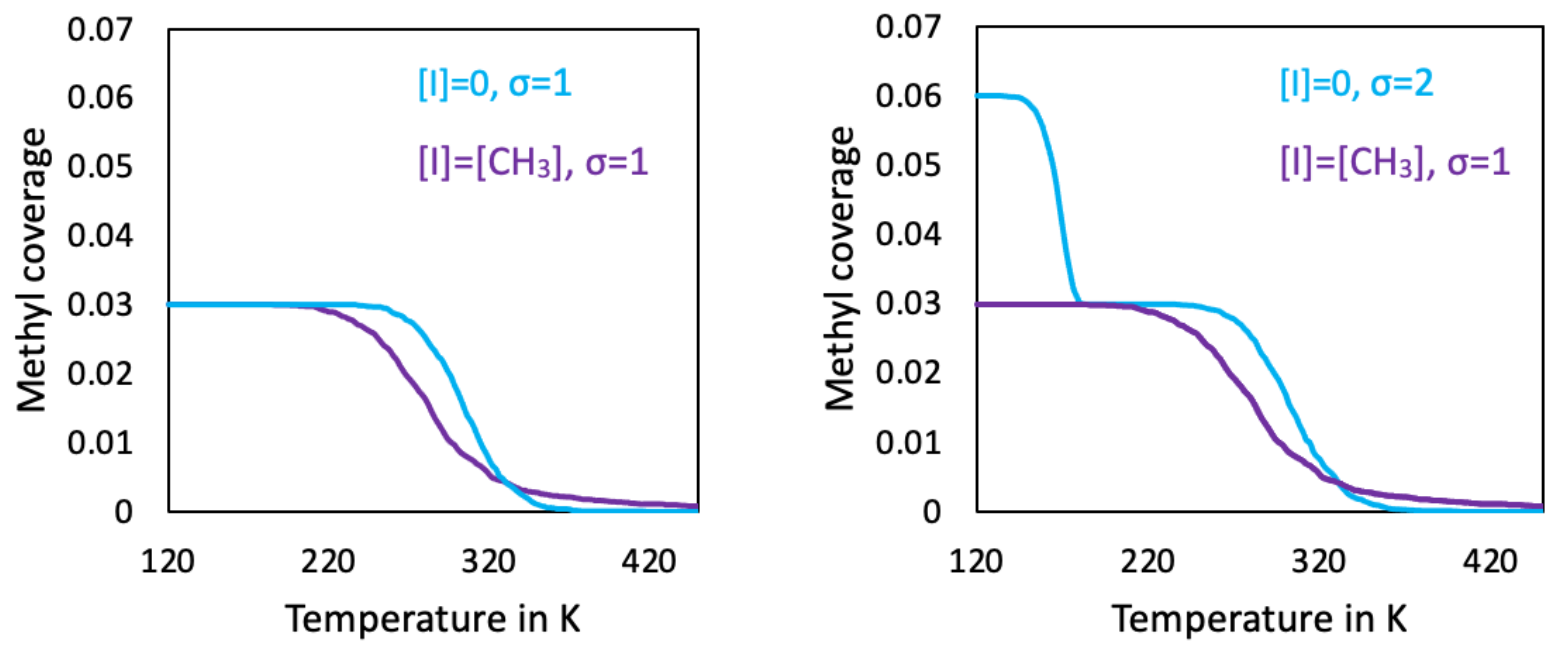

Figure S2. Methyl coverages during the simulated TPD experiments on NiAu SAA. The coverage is defined as the number of methyl groups normalized by the number of top Au sites. 